\title{
Pseudo-Kähler-Einstein geometries
}

\author{
Carlos G. Boiza@ and Jose A. R. Cembranos® \\ Departamento de Física Teórica and IPARCOS, Facultad de Ciencias Físicas, \\ Universidad Complutense de Madrid, Plaza de Ciencias, n 1, 28040 Madrid, Spain
}

(Received 17 December 2021; accepted 16 February 2022; published 8 March 2022)

\begin{abstract}
Solutions to vacuum Einstein field equations with cosmological constants, such as the de Sitter space and the anti-de Sitter space, are basic in different cosmological and theoretical developments. It is also well known that complex structures admit metrics of this type. The most famous example is the complex projective space endowed with the Fubini-Study metric. In this work, we perform a systematic study of Einstein complex geometries derived from a logarithmic Kähler potential. Depending on the different contribution to the argument of such logarithmic term, we shall distinguish among direct, inverted and hybrid coordinates. They are directly related to the signature of the metric and determine the maximum domain of the complex space where the geometry can be defined.
\end{abstract}

DOI: 10.1103/PhysRevD.105.065006

\section{INTRODUCTION}

Complex manifolds have been implemented in modern theories, mainly within string frameworks. For example, the bosonic string theory is formulated on a two-dimensional differentiable manifold which is embedded on the $d$-dimensional Minkowskian space. The action of this theory is the Polyakov action [1], and it is invariant under diffeomorphisms and Weyl transformations. When the theory is quantized via path integral, we must take into account the overcounting due to the symmetries, and, for this purpose, it is possible to fix the gauge by taking the called unit gauge, which fixes the metric to be the unit one, but there is still a residual symmetry due to conformal transformations, so we are dealing with a two-dimensional conformal field theory. Two-dimensional differentiable manifolds with transition functions being conformal are isomorphic to complex manifolds of dimension one (Riemann surfaces), so the theory can be formulated on a one-dimensional complex manifold.

It is interesting to implement the concept of complex manifolds within the structure of space-time. Again, the better known example of this can be found in superstring theory where there are six extra dimensions compactified on Calabi-Yau manifolds [2], which are a specific kind of Kähler complex manifold.

In this work, we are particularly interested in the implementation of the concepts of complex differential geometry on extensible (not compactified) space-time structures.

Published by the American Physical Society under the terms of the Creative Commons Attribution 4.0 International license. Further distribution of this work must maintain attribution to the author(s) and the published article's title, journal citation, and DOI. Funded by SCOAP ${ }^{3}$.
Within real manifolds, it is well known the existence of solutions of the vacuum Einstein field equations with cosmological constant. These types of manifolds are called Einstein manifolds. All constant sectional curvature manifolds are of this kind. Examples of Riemannian manifolds with constant sectional curvature are the usual Euclidean space of arbitrary dimensions with the usual Euclidean metric (which is flat), and the unit $n$-sphere with the round metric (which has positive curvature). The most important examples of pseudo-Riemannian manifolds with constant sectional curvature are the de Sitter space $[3,4]$ (of positive curvature), which serves as a simple model of an accelerated expanding universe, and the anti-de Sitter space (of negative curvature), which recently acquired relevance in string theory due to the AdS/CFT correspondence proposed by Maldacena [5].

There are also well-known examples of complex Einstein manifolds. In the case of positive-definite signatures and constant bisectional curvature, we can distinguish among the complex projective space with the Fubini-Study (FS) metric (which has positive curvature), the Euclidean complex space (flat), and the unit ball (negative curvature). All this manifolds are Kähler manifolds.

The main objective of this work is to perform a systematic classification of complex Einstein geometries derived from a logarithmic Kähler potential. We will generalize the study to non-positive-definite metrics, i.e., we will analyze pseudo-Kähler geometries and analyze their main curvature properties.

\section{REVIEW OF BASIC CONCEPTS}

First of all, we will summarize the main basic concepts of complex differential geometry in order to clarify the notations and conventions we will use throughout the work. 


\section{A. Complex manifolds}

An $m$ dimensional differentiable manifold $M$ is a topological space which can be covered by a family of open subsets $\left\{U_{i}\right\}\left(M=\cup_{i} U_{i}\right)$, where each $U_{i}$ is homeomorphic to an open subset $U_{i}^{\prime}$ of $\mathbb{R}^{m}$ : there is a map $\psi_{i}: U_{i} \subset M \rightarrow U_{i}^{\prime} \subset \mathbb{R}^{m}$ which is a homeomorphism. It is also required differentiability of the maps $\Psi_{i j}=\psi_{i} \circ \psi_{j}^{-1}$ in regions where $U_{i} \cap U_{j} \neq \varnothing$. To define a complex manifold we now require open subsets $U_{i}$ 's to be homeomorphic to open subsets $U_{i}^{\prime}$ 's of $\mathbb{C}^{m}$ (there is a map $\psi_{i}: U_{i} \subset M \rightarrow$ $U_{i}^{\prime} \subset \mathbb{C}^{m}$ which is a homeomorphism for each $U_{i}$ ) and maps $\Psi_{i j}=\psi_{i} \circ \psi_{j}^{-1}$ in regions $U_{i} \cap U_{j} \neq \varnothing$ to be holomorphic [6]. The dimension $m$ is now understood as the complex dimension of the manifold. Note that all complex manifolds are differentiable manifolds because holomorphicity of maps automatically implies differentiability, so an $m$ dimensional complex manifold can also be seen as a $2 m$ dimensional differentiable manifold. It does not happen the same in reverse order because differentiability does not necessarily imply holomorphicity, so not all differentiable manifolds are complex ones.

Since a complex manifold is a differentiable one, all the machinery developed for differentiable ones (vectors, forms, tensors, tensor fields, ...) can be used in a complex manifold. Consider a differentiable manifold which admits a tensor field $J$ of type $(1,1)$ such that, at each point $p \in M$, it squares to minus the identity $J_{p}^{2}=-i d_{T_{p} M}$; this is called an almost complex manifold and the associated tensor field $J$ its almost complex structure [7]. Define the Nijenhuis tensor field $N: T M \times T M \rightarrow T M$ as the tensor which acting on two vector fields $v, w \in T M$ gives:

$$
N(v, w)=[v, w]+J[J v, w]+J[v, J w]-[J v, J w] .
$$

It was shown by Newlander and Nirenberg [8] that the necessary and sufficient condition for a manifold to be a complex one is the vanishing of the Nijenhuis tensor $N=0$; in this case we call $J$ the complex structure of the manifold.

\section{B. Complexifications}

Consider a differentiable manifold $M$. The set of smooth functions $\mathbb{F}(M)$ is complexified [6] in the following way: take two functions $g, h \in \mathbb{F}(M)$ and form a new one $f=g+i h$, which is now a complex valued function $f: M \rightarrow \mathbb{C}$; the set of functions constructed in this way forms what is called the complexification of $\mathbb{F}(M)$, denoted by $\mathbb{E}(M)^{\mathbb{C}}$. Similarly, the tangent space of $M$ at the point $p \in M$ can be complexified: take two vectors $v, w \in T_{p} M$ and form a new one $u=v+i w$; the set of vectors constructed in this way forms the complexified tangent space $T_{p} M^{\mathbb{C}}$ (note that this is a vector space under the well defined complex addition and complex scalar multiplication). Vector fields are complexified in a similar way.
A linear operator $A$ acting on the vector space $T_{p} M$ is extended to act on the complexified one as $A(u)=$ $A(v+i w)=A(v)+i A(w)$, where $u=v+i w \in T_{p} M^{\mathbb{C}}$. All tensors and tensor fields can be analogously complexified. Note that complexified vector spaces have same dimension as ordinary ones.

Since an $m$ dimensional complex manifold can be seen as a $2 m$ differentiable one [6], we can consider a local coordinate basis of the tangent space at $p \in M$ given by $2 m$ vectors $\left\{\partial / \partial x^{1}, \ldots, \partial / \partial x^{m}, \partial / \partial y^{1}, \ldots, \partial / \partial y^{m}\right\}$ associated to the real part and the imaginary part of complex coordinates $z^{\mu}=x^{\mu}+i y^{\mu}$. Its dual space is spanned by $\left\{d x^{1}, \ldots, d x^{m}, d y^{1}, \ldots, d y^{m}\right\}$. In this basis, the complex structure $J$ acts on vectors as $J_{p}\left(\partial / \partial x^{\mu}\right)=\partial / \partial y^{\mu}$, $J_{p}\left(\partial / \partial y^{\mu}\right)=-\partial / \partial x^{\mu}$. Complexifying the tangent space, we can define a new basis given by $\partial / \partial z^{\mu}=$ $\left(\partial / \partial x^{\mu}-i \partial / \partial y^{\mu}\right) / 2, \partial / \partial \bar{z}^{\mu}=\left(\partial / \partial x^{\mu}+i \partial / \partial y^{\mu}\right) / 2$. The dual basis is given by $d z^{\mu}=d x^{\mu}+i d y^{\mu}, d \bar{z}^{\mu}=d x^{\mu}-i d y^{\mu}$. In this basis, $J$ acts as $J_{p}\left(\partial / \partial z^{\mu}\right)=i \partial / \partial z^{\mu}, J_{p}\left(\partial / \partial \bar{z}^{\mu}\right)=$ $-i \partial / \partial \bar{z}^{\mu}$.

\section{Pseudo-Kähler manifolds}

Consider a complex manifold $M$ and a Riemannian metric $g$ defined on $M$ as a differentiable manifold. Its action can be generalized to the complexified tangent space at a point $p \in M$ expressed by $T_{p} M^{\mathbb{C}}$ [6]. With this, it is possible to calculate the components of the metric with respect to the complexified basis $g_{p, \mu \nu}=g_{p}\left(\partial / \partial z^{\mu}, \partial / \partial z^{\nu}\right)$, $g_{p, \mu \bar{\nu}}=g_{p}\left(\partial / \partial z^{\mu} \partial / \partial \bar{z}^{\nu}\right), g_{p, \bar{\mu} \nu}=g_{p}\left(\partial / \partial \bar{z}^{\mu}, \partial / \partial z^{\nu}\right), g_{p, \bar{\mu} \bar{\nu}}=$ $g_{p}\left(\partial / \partial \bar{z}^{\mu}, \partial / \partial \bar{z}^{\nu}\right)$. If the metric $g$ satisfies $g_{p}\left(J_{p} v, J_{p} w\right)=$ $g_{p}(v, w)$ at each point $p \in M$, where $J$ is the complex structure and $v, w \in T_{p} M$, it is said to be a Hermitian metric [7] and the manifold a Hermitian manifold. For a Hermitian metric, only components $g_{\mu \bar{\nu}}, g_{\bar{\mu} \nu}$ are not null: $g_{\mu \nu}=g_{\bar{\mu} \bar{\nu}}=0$. We define the Kähler form $\omega$ of a Hermitian metric $g$ as a tensor field whose action on vectors of the tangent space at a point $p \in M$ is given by $\omega_{p}(v, w)=g_{p}\left(J_{p} v, w\right)$, where $v, w \in T_{p} M$; the Kähler form is antisymmetric, so it defines a two-form. We say a metric is a Kähler metric if its Kähler form is closed: $d \omega=0$. If a metric is Kähler, the manifold, where it is defined on, is called a Kähler manifold. Consider a complex manifold $M$ and a Hermitian metric $g$ on it; the necessary and sufficient condition for the metric $g$ to be a Kähler one is $\nabla_{l c} J=0$ [7], where $J$ is the complex structure and $\nabla_{l c}$ is the Levi-Civita connection (note that $\nabla_{l c}$ is well defined since a complex manifold is a differentiable one and uniquely determined by $g$, which is Riemannian by definition). A Kähler metric can always be locally expressed as $g_{\mu \bar{\nu}}=\partial_{\mu} \partial_{\bar{\nu}} K_{i}$ [9], where $K_{i} \in \mathbb{F}\left(U_{i}\right)$ is called the Kähler potential and $U_{i}$ is an open subset of $M$, and $\partial_{\mu}, \partial_{\bar{\nu}}$ are partial derivatives with respect local coordinates $z^{\mu}, \bar{z}^{\nu}$, respectively. It is required $K_{i}$ to transform as $K_{j}(w, \bar{w})=K_{i}(z, \bar{z})+$ $\phi_{i j}(z)+\psi_{i j}(\bar{z})$ in regions where $U_{i} \cap U_{j} \neq \varnothing$, with $\phi$ 
holomorphic and $\psi$ antiholomorphic, and where $w$ are complex local coordinates of $U_{j}$ and $z$ are complex local coordinates of $U_{i}$; by this way, the metric $g$ transforms properly under general coordinate transformations. Reciprocally, all metrics expressed as the double derivative $\partial \bar{\partial}$ of a Kähler potential are Kähler. Similar definitions and conclusions are obtained relaxing the positive-definite condition of the metric and considering now a pseudoRiemannian one. In this way, we will talk about pseudoKähler metrics.

Since through this work we deal with metrics which will be shown to be all Kähler or pseudo-Kähler, we will often not make distinctions between them and their associated Kähler or pseudo-Kähler forms. We will use the same notation for both and we will refer to them indistinguishably.

\section{Curvature of pseudo-Kähler metrics}

Consider a Kähler metric $g$ on a Kähler manifold $M$. We can define a connection acting on the complexified tangent space as $\nabla_{c h}: T M^{\mathbb{C}} \times T M^{\mathbb{C}} \rightarrow T M^{\mathbb{C}}$, and being compatible with the complex structure $\nabla_{c h} J=0$ [7]. In local coordinates, we define the Christoffel symbols of the connection as:

$$
\begin{aligned}
& \nabla_{c h, \mu} \frac{\partial}{\partial z^{\nu}}=\Gamma_{\mu \nu}^{\lambda} \frac{\partial}{\partial z^{\lambda}}+\Gamma_{\mu \nu}^{\bar{\lambda}} \frac{\partial}{\partial \bar{z}^{\lambda}}, \\
& \nabla_{c h, \mu} \frac{\partial}{\partial \bar{z}^{\nu}}=\Gamma_{\mu \bar{\nu}}^{\lambda} \frac{\partial}{\partial z^{\lambda}}+\Gamma_{\mu \bar{\nu}}^{\bar{\lambda}} \frac{\partial}{\partial \bar{z}^{\lambda}} .
\end{aligned}
$$

For the connection to be compatible with the complex structure $\nabla_{c h} J=0$, it is required to vanish all Christoffel symbols except $\Gamma_{\mu \nu}^{\lambda}, \Gamma_{\bar{\mu} \bar{\nu}}^{\bar{\lambda}}=\overline{\Gamma_{\mu \nu}^{\lambda}}$. In addition, by requiring metric compatibility $\nabla_{c h} g=0$, they result to be totally determined by the metric:

$$
\Gamma_{\mu \nu}^{\lambda}=g^{\lambda \bar{\rho}} \frac{\partial g_{\nu \bar{\rho}}}{\partial z^{\mu}} .
$$

This is called the Chern connection [10], and can be defined similarly on Hermitian manifolds (not necessarily Kähler manifolds), but, for Kähler manifolds, and only for Kähler manifolds [11], the Chern connection is the same as the Levi-Civita one extended to act on $T M^{\mathbb{C}} \times T M^{\mathbb{C}}$.

The components of the Riemann tensor associated to the Hermitian connection [12,13], in local complex coordinates, are given by:

$$
R_{\mu \bar{\nu} \rho \bar{\sigma}}=-\frac{\partial^{2} g_{\mu \bar{\nu}}}{\partial z^{\rho} \partial \bar{z}^{\sigma}}+g^{\lambda \bar{\eta}} \frac{\partial g_{\lambda \bar{\nu}}}{\partial z^{\rho}} \frac{\partial g_{\mu \bar{\eta}}}{\partial \bar{z}^{\sigma}}
$$

and, by taking the trace in two first components, we obtain the expression for the components of a new tensor (known as Ricci tensor) in local coordinates:

$$
\operatorname{Ric}_{\rho \bar{\sigma}}=g^{\mu \bar{\nu}} R_{\mu \bar{\nu} \rho \bar{\sigma}}=-\frac{\partial^{2}}{\partial z^{\rho} \partial \bar{z}^{\sigma}}(\log |g|),
$$

where $g$ is the determinant of the metric.

We say that a Kähler manifold $M$ is of constant bisectional curvature [7] if the components in local coordinates of the Riemann tensor associated to the Hermitian connection can be written in terms of the Kähler metric ones as $R_{\mu \bar{\nu} \rho \bar{\sigma}}=\lambda_{c}\left(g_{\mu \bar{\nu}} g_{\rho \bar{\sigma}}+g_{\mu \bar{\sigma}} g_{\rho \bar{\nu}}\right)$, where $\lambda_{c}$ is a real constant. For $\lambda_{c}>0$, the bisectional curvature is said to be positive, for $\lambda_{c}=0$, null, and for $\lambda_{c}<0$, negative.

We say that a Kähler metric $g$ is a Kähler-Einstein metric [7] if the Ricci tensor obtained as the trace of the Riemann tensor associated to the Hermitian connection is proportional to the metric; in local coordinates $R i c_{\mu \bar{\nu}}=\lambda_{r} g_{\mu \bar{\nu}}$, where $\lambda_{r}$ is a real constant.

The same concepts summarized in this section are straightforward generalized to pseudo-Hermitian metrics.

\section{E. Fubini-Study metric}

Let us consider the space $N$ given by:

$$
N=\left\{Z \in \mathbb{C}^{n+1}, Z \neq 0\right\} / \sim,
$$

where we identify $Z, W \in \mathbb{C}^{n+1}$ by $Z \sim W$ if there exist a complex number $c \neq 0$ such that $W=c Z$. This is called the complex projective space and it is an example of a complex manifold of dimension $n$. This manifold is isomorphic to the quotient space given by $S^{2 n+1} / U(1)$, where $S^{2 n+1}$ is the unit $2 n+1$ hypersphere in $\mathbb{C}^{n+1}$ and $U(1)$ is the Abelian unitary group. The unit sphere can be written in terms of complex coordinates $Z$ as:

$$
S^{2 n+1}=\left\{Z \in \mathbb{C}^{n+1}: \sum_{i=1}^{n+1}\left|Z_{i}\right|^{2}=1\right\},
$$

and the quotient space identified with the manifold $N=$ $S^{2 n+1} / U(1)$ can be completely covered by the union of sets $\cup_{j} U_{j}$, where $U_{j}$ is given by:

$$
U_{j}\left(Z_{j} \neq 0\right)=\left\{z \in \mathbb{C}^{n}: \sum_{\substack{i=1 \\ i \neq j}}^{n+1}\left|z_{i}\right|^{2}>0\right\},
$$

where we have defined new coordinates $z_{i}=Z_{i} / Z_{j}$.

We can now consider the Hermitian metric $g$ on $C^{n+1}$ given by $g=\sum_{i=1}^{n+1}\left|d Z_{i}\right|^{2}$ and calculate the induced one by the map $\pi: M(Z) \rightarrow N(z)$. The result is the FS metric, which is given by: 


$$
g_{f s, \mu \bar{\nu}}=\partial_{\mu} \partial_{\bar{\nu}} \log \left(1+\sum_{\substack{i=1 \\ i \neq j}}^{n+1}\left|z_{i}\right|^{2}\right) .
$$

We can write the metric $g_{f s}$ in its matrix form (assuming, for instance, $j=n+1$ ):

$$
g_{f s}=\frac{1}{K_{f s}^{2}}\left(\begin{array}{cccc}
K_{f s}-\left|z_{1}\right|^{2} & -\bar{z}_{1} z_{2} & \cdots & -\bar{z}_{1} z_{n} \\
-z_{1} \bar{z}_{2} & K_{f s}-\left|z_{2}\right|^{2} & \ldots & -\bar{z}_{2} z_{n} \\
\vdots & \vdots & \ddots & \vdots \\
-z_{1} \bar{z}_{n} & -z_{2} \bar{z}_{n} & \cdots & K_{f s}-\left|z_{n}\right|^{2}
\end{array}\right) \text {, }
$$

where $K_{f s}=1+\sum_{\substack{i=1 \\ i \neq j}}^{n+1}\left|z_{i}\right|^{2}$, and, since it is a Hermitian and positive-definite matrix, the metric is Hermitian. This metric is also a Kähler metric, since it is Hermitian and can be written as the double derivative $\partial \bar{\partial}$ of the potential $\log K_{f s}$. We can also calculate its determinant: $\operatorname{det} g_{f s}=1 / K_{f s}^{n+1}$.

The determinant allows to compute all the components of the Ricci tensor by using Eq. (6). The calculation shows that this metric is a Kähler-Einstein metric with $\lambda_{r}=n+1$ as defined in Sec. II D: $R_{\mu \bar{\nu}}=(n+1) g_{f s, \mu \bar{\nu}}$, where $n$ is the dimension of the complex manifold $N$.

The group $S U(n+1)$ generates isometries of the FS metric $g_{f s}$ and also acts transitively on the complex projective space $N$. Taking this fact into account, it is enough to show the that the bisectional curvature is constant at the point $z=0$, to prove that it is constant at any point. At $z=0$, the calculus of the components of the Riemann tensor (5) simplifies because all first derivatives of this metric vanish at this point. In this way, it is easy to show that $\left.R_{\mu \bar{\nu} \rho \bar{\sigma}}\right|_{z=0}=\left.\left(g_{f s, \mu \bar{\nu}} g_{f s, \rho \bar{\sigma}}+g_{f s, \mu \bar{\sigma}} g_{f s, \rho \bar{\nu}}\right)\right|_{z=0}$. Therefore, we have a positive constant bisectional curvature with $\lambda_{c}=1$ as defined in Sec. II D.

\section{F. Unit ball}

Consider the space $M$ given by:

$$
M^{B}=\left\{Z \in \mathbb{C}^{n+1}:-\left|Z_{0}\right|^{2}+\sum_{i=1}^{n}\left|Z_{i}\right|^{2}=-1\right\} .
$$

We note that $Z_{0} \neq 0$ because $\left|Z_{0}\right|^{2}=1+\sum_{i=1}^{n}\left|Z_{i}\right|^{2}>0$. With this, the $n$ dimensional complex manifold given by $N^{B}=M^{B} / U(1)$ can be identified with the open unit ball in $\mathbb{C}^{n}[14]:$

$$
N^{B}=\left\{z \in \mathbb{C}^{n}: \sum_{i=1}^{n}\left|z_{i}\right|^{2}<1\right\}
$$

where we have defined new coordinates $z_{i}=Z_{i} / Z_{0}$. The map $\pi^{B}: M^{B}(Z) \rightarrow N^{B}(z)$ induces, from the Hermitian metric $g=-\left|d Z_{0}\right|^{2}+\sum_{i=1}^{n}\left|d Z_{i}\right|^{2}$, a new metric on $N^{B}$ given by:

$$
g_{b, \mu \bar{\nu}}=-\partial_{\mu} \partial_{\bar{\nu}} \log \left(1-\sum_{i=1}^{n}\left|z_{i}\right|^{2}\right) .
$$

The matrix form of $g_{b}$ is given by:

$$
g_{b}=\frac{1}{K_{b}^{2}}\left(\begin{array}{cccc}
K_{b}+\left|z_{1}\right|^{2} & \bar{z}_{1} z_{2} & \cdots & \bar{z}_{1} z_{n} \\
z_{1} \bar{z}_{2} & K_{b}+\left|z_{2}\right|^{2} & \cdots & \bar{z}_{2} z_{n} \\
\vdots & \vdots & \ddots & \vdots \\
z_{1} \bar{z}_{n} & z_{2} \bar{z}_{n} & \cdots & K_{b}+\left|z_{n}\right|^{2}
\end{array}\right) \text {, }
$$

where $K_{b}=1-\sum_{i=1}^{n}\left|z_{i}\right|^{2}$.

The analysis of this new metric is similar to the FS one, but we now obtain a negative constant bisectional curvature with $\lambda_{r}=-(n+1), \lambda_{c}=-1$, i.e., $R_{\mu \bar{\nu}}=-(n+1) g_{b, \mu \bar{\nu}}$, and $R_{\mu \bar{\nu} \rho \bar{\sigma}}=-\left(g_{b, \mu \bar{\nu}} g_{b, \rho \bar{\sigma}}+g_{b, \mu \bar{\sigma}} g_{b, \rho \bar{\nu}}\right)$.

\section{ONE NONDIRECT COORDINATE}

We begin to study the simplest case in which we introduce a single nondirect coordinate. Firstly, we will consider the introduction of an inverted coordinate and, secondly, the introduction of an hybrid coordinate.

\section{A. One inverted coordinate}

As mentioned in Sec. II E, the $n$ dimensional complex projective space is isomorphic to the quotient space between the real unit $2 n+1$ hypersphere and the Abelian unitary group $U(1)$. This allows us to obtain the FS metric as the induced metric of the Euclidian Hermitian metric in $\mathbb{C}^{n+1}$ dimensions on the quotient space.

The FS metric is generated by the logarithmic Kähler potential introduced in Eq. (10). We will use the potential in order to classify the different coordinates. For the FS metric, all coordinates are direct since the square of their real and imaginary parts contribute positively to the argument of the logarithmic potential. We can study the effect of introducing an inverted coordinate, i.e., a coordinate whose square of its real and imaginary parts contribute negatively to the logarithmic Kähler potential:

$$
\mathcal{G}_{\mu \bar{\nu}}=\partial_{\mu} \partial_{\bar{\nu}} \log \left(1-\left|z_{0}\right|^{2}+\sum_{\substack{i=1 \\ i \neq j}}^{n}\left|z_{i}\right|^{2}\right) .
$$

Firstly, we note that this metric can be obtained as the induced of a Hermitian one in $\mathbb{C}^{n+1}$ dimensions on a quotient space, similar to the FS case. Let as consider the space $M^{I}$ given by: 


$$
M^{I}=\left\{Z \in \mathbb{C}^{n+1}:-\left|Z_{0}\right|^{2}+\sum_{i=1}^{n}\left|Z_{i}\right|^{2}=1\right\} .
$$

We note that $\exists i \in[1, n]: Z_{i} \neq 0$ because $\sum_{i=1}^{n}\left|Z_{i}\right|^{2}=$ $1+\left|Z_{0}\right|^{2}>0$. We now consider the quotient space given by $N^{I}=M^{I} / U(1)$; this is an $n$ dimensional complex manifold. From the observation just made, we conclude that all $N$ can be covered by the union of sets $\cup_{j} U_{j}$, where $U_{j}$ is given by:

$U_{j}\left(Z_{j} \neq 0\right)=\left\{z \in \mathbb{C}^{n}:-\left|z_{0}\right|^{2}+\sum_{\substack{i=1 \\ i \neq j}}^{n}\left|z_{i}\right|^{2}>-1\right\}$,

where we have defined new coordinates $z_{0}=Z_{0} / Z_{j}$, $z_{i}=Z_{i} / Z_{j}$. The map $\pi^{I}: M^{I}(Z) \rightarrow N^{I}(z)$ induces, from the Hermitian metric $g=-\left|d Z_{0}\right|^{2}+\sum_{i=1}^{n}\left|d Z_{i}\right|^{2}$, the metric introduced in Eq. (16).

Writing the metric $\mathcal{G}$ in its matrix form (assuming, for instance, $j=n$ ):

$\mathcal{G}=\frac{1}{G^{2}}\left(\begin{array}{cccc}-G-\left|z_{0}\right|^{2} & \bar{z}_{0} z_{1} & \cdots & \bar{z}_{0} z_{n-1} \\ z_{0} \bar{z}_{1} & G-\left|z_{1}\right|^{2} & \cdots & -\bar{z}_{1} z_{n-1} \\ \vdots & \vdots & \ddots & \vdots \\ z_{0} \bar{z}_{n-1} & -z_{1} \bar{z}_{n-1} & \cdots & G-\left|z_{n-1}\right|^{2}\end{array}\right)$,

where $G=1-\left|z_{0}\right|^{2}+\sum_{\substack{i=1 \\ i \neq j}}^{n}\left|z_{i}\right|^{2}$, we see it is a pseudoHermitian metric since this is a Hermitian matrix and we have lost the positive-definite condition having introduced one inverted coordinate. We can also write it as a $\partial \bar{\partial}$ derivative of the potential $\log G$, so it is a closed form $(d \mathcal{G}=0)$. With this, we have found the metric $\mathcal{G}$ to be a pseudo-Kähler one.

It is easy to compute its determinant: $\operatorname{det} \mathcal{G}=-1 / G^{n+1}$ and the Ricci curvature in a similar way to the FS case. In fact, the components $R i c_{k l}$ are proportional to the metric: Ric $=(n+1) \mathcal{G}$. So we conclude that $\mathcal{G}$ is a pseudo-KählerEinstein metric.

Finally, we will discuss the bisectional curvature. We will find it to be a constant bisectional curvature one. For this to be shown, we can use similar arguments as in FS case. The indefinite special unitary group $S U(1, n)$ is the set of matrices acting on $\mathbb{C}^{n+1}$ which preserve the Hermitian form $g(W, Z)=-\overline{W_{0}} Z_{0}+\sum_{i=1}^{n} \overline{W_{i}} Z_{i}$, where $W, Z \in \mathbb{C}^{n+1}$, with determinant one. This group acts on $N$ as an isometry and transitively, so it is enough to show the relation between Riemann curvature and metric at $z=0$. Calculus is analogous to FS one and we finally obtain the same relation $\left.R_{i \bar{j} k \bar{l}}\right|_{z=0}=\left.\left(\mathcal{G}_{i \bar{j}} \mathcal{G}_{k \bar{l}}+\mathcal{G}_{i \bar{l}} \mathcal{G}_{k \bar{j}}\right)\right|_{z=0}$, so this metric is also of positive constant bisectional curvature with $\lambda_{c}=1$. We note that the pseudo-Kähler-Einstein condition could have been gotten directly from this last relation of positive constant bisectional curvature taking a trace in both sides, but we have used the first method of calculating the determinant because in the next sections it will be important to use separate methods while both conditions are not necessarily satisfied at the same time (a metric to be Einstein is not implied to be of constant bisectional curvature).

It is also interesting to note the analogy between the space $N^{I}$ and the real de Sitter space. This has been emphasized by naming $N^{I}$ as the $n$ dimensional complex de Sitter space [15].

\section{B. One hybrid coordinate}

In the metric $\mathcal{G}(16)$, we introduced an inverted coordinate, so its contribution to the argument of the logarithmic potential was $-|z|^{2}=-\mathfrak{R e}(z)^{2}-\mathfrak{I m}(z)^{2}$, where both parts (real and imaginary) contributed negatively. In this section, we shall consider a mixed contribution. We only reverse the sign of one of the parts (the imaginary one, for example). Therefore, we introduce an hybrid coordinate whose contribution is given by $\left(z^{2}+\bar{z}^{2}\right) / 2=$ $\mathfrak{R e}(z)^{2}-\mathfrak{I m}(z)^{2}$. The new metric can be written as:

$$
\mathcal{H}_{\mu \bar{\nu}}=\partial_{\mu} \partial_{\bar{\nu}} \log \left[1+\frac{1}{2}\left(z_{1}^{2}+\bar{z}_{1}^{2}\right)+\sum_{i=2}^{n}\left|z_{i}\right|^{2}\right] .
$$

The corresponding matrix form is:

$$
\mathcal{H}=\frac{1}{H^{2}}\left(\begin{array}{cccc}
-\left|z_{1}\right|^{2} & -z_{1} z_{2} & \cdots & -z_{1} z_{n} \\
-\overline{z_{1} z_{2}} & H-\left|z_{2}\right|^{2} & \cdots & -\bar{z}_{2} z_{n} \\
\vdots & \vdots & \ddots & \vdots \\
-\overline{z_{1} z_{n}} & -z_{2} \bar{z}_{n} & \cdots & H-\left|z_{n}\right|^{2}
\end{array}\right),
$$

where $H=1+\frac{1}{2}\left(z_{1}^{2}+\bar{z}_{1}^{2}\right)+\sum_{i=2}^{n}\left|z_{i}\right|^{2}$. Again, we can see that $\mathcal{H}$ is a pseudo-Kähler metric. It is pseudoHermitian since its matrix form is Hermitian but the positive-definite condition is not verified by the introduction of the hybrid coordinate. It is a closed form $(d \mathcal{H}=0)$ because we can write it as the $\partial \bar{\partial}$ derivative of the potential $\log H$.

The determinant of this metric is also straightforward: $\operatorname{det} \mathcal{H}=-\left|z_{1}\right|^{2} / H^{n+1}$. And it can be used to compute the Ricci tensor by taking into account Eq. (6). We conclude that the Ricci form is proportional to the metric: $\lambda_{r}=n+1: \quad \operatorname{Ric}=(n+1) \mathcal{H}$, so this metric is also pseudo-Kähler-Einstein type. However, this metric does not have associated a constant bisectional curvature. In order to prove it, it is enough to compute the Riemann tensor in one given subspace where the constant bisectional curvature condition is not verified. This is particularly simple in the subspace given by $z_{i}=0 \forall i \in[2, n]$, for the components with $\mu \in[2, n]$. 


\section{GENERALIZATION TO AN ARBITRARY NUMBER OF NONDIRECT COORDINATES}

In this section we shall study the most general geometries that can be built from a Käler logarithmic potential $F$ with an arbitrary number of direct, inverted and hybrid coordinates.

\section{A. Hybrid coordinates condition}

Let us consider a form given by $\mathcal{F}_{\mu \bar{\nu}}=\partial_{\mu} \partial_{\bar{\nu}} \log F$, where:

$F=1+\sum_{u=1}^{l} \frac{1}{2}\left(z_{u}^{2}+\bar{z}_{u}^{2}\right)+\sum_{v=l+1}^{l+m}\left|z_{v}\right|^{2}-\sum_{w=l+m+1}^{l+m+p}\left|z_{w}\right|^{2}$,

and $l, m, p$ sum up to $n$ general dimensions: $l+m+p=n$. From now on, we will use letters $u, v$, $w$ for complex coordinates $z_{u}, z_{v}, z_{w}$, respectively. So we can replace subindices $v=l+i, w=l+m+j$ with $i, j$, respectively, for $i \in[1, m], j \in[1, p]$, simplifying the notation. In the most general matrix form will appear nine distinguishable blocks. Let us call them $\mathbf{1}, \mathbf{2}, \ldots, 9$ :

$$
\mathcal{F}=\frac{1}{F^{2}}\left(\begin{array}{lll}
1 & 2 & 3 \\
4 & 5 & 6 \\
7 & 8 & 9
\end{array}\right)
$$

The main diagonal blocks $\mathbf{1}, \mathbf{5 , 9}$ are the nonmixing terms, in the sense that they are obtained acting on the potential with derivatives which do not mix different types $(u, v, w)$ of complex coordinates. Therefore, they are three different square blocks: operators $\partial_{u} \partial_{\bar{u}}, \partial_{v} \partial_{\bar{v}}, \partial_{w} \partial_{\bar{w}}$ generate blocks $\mathbf{1 , 5}, \mathbf{9}$, respectively. Explicitly, they are given by:

$$
\begin{gathered}
\mathbf{1}=\left(\begin{array}{cccc}
-\left|u_{1}\right|^{2} & -u_{1} \bar{u}_{2} & \cdots & -u_{1} \bar{u}_{l} \\
-\bar{u}_{1} u_{2} & -\left|u_{2}\right|^{2} & \cdots & -u_{2} \bar{u}_{l} \\
\vdots & \vdots & \ddots & \vdots \\
-\bar{u}_{1} u_{l} & -\bar{u}_{2} u_{l} & \cdots & -\left|u_{l}\right|^{2}
\end{array}\right), \\
\mathbf{5}=\left(\begin{array}{cccc}
F-\left|v_{1}\right|^{2} & -\bar{v}_{1} v_{2} & \cdots & -\bar{v}_{1} v_{m} \\
-v_{1} \bar{v}_{2} & F-\left|v_{2}\right|^{2} & \cdots & -\bar{v}_{2} v_{m} \\
\vdots & \vdots & \ddots & \vdots \\
-v_{1} \bar{v}_{m} & -v_{2} \bar{v}_{m} & \cdots & F-\left|v_{m}\right|^{2}
\end{array}\right), \\
\mathbf{9}=\left(\begin{array}{cccc}
-F-\left|w_{1}\right|^{2} & -\bar{w}_{1} w_{2} & \cdots & -\bar{w}_{1} w_{p} \\
-w_{1} \bar{w}_{2} & -F-\left|w_{2}\right|^{2} & \cdots & -\bar{w}_{2} w_{p} \\
\vdots & \vdots & \ddots & \vdots \\
-w_{1} \bar{w}_{p} & -w_{2} \bar{w}_{p} & \cdots & -F-\left|w_{p}\right|^{2}
\end{array}\right) .
\end{gathered}
$$

All other blocks mix different types of complex coordinates, so they are not generally square. Blocks $\mathbf{2 , 4}$ are generated by $\partial_{u} \partial_{\bar{v}}, \partial_{v} \partial_{\bar{u}}$, respectively, and are given by:

$$
\begin{gathered}
\mathbf{2}=\left(\begin{array}{cccc}
-u_{1} v_{1} & -u_{1} v_{2} & \cdots & -u_{1} v_{m} \\
-u_{2} v_{1} & -u_{2} v_{2} & \cdots & -u_{2} v_{m} \\
\vdots & \vdots & \ddots & \vdots \\
-u_{l} v_{1} & -u_{l} v_{2} & \cdots & -u_{l} v_{m}
\end{array}\right), \\
\mathbf{4}=\left(\begin{array}{cccc}
-\overline{u_{1} v_{1}} & -\overline{u_{2} v_{1}} & \cdots & -\overline{u_{l} v_{1}} \\
-\overline{u_{1} v_{2}} & -\overline{u_{2} v_{2}} & \cdots & -\overline{u_{l} v_{2}} \\
\vdots & \vdots & \ddots & \vdots \\
-\overline{u_{1} v_{m}} & -\overline{u_{2} v_{m}} & \cdots & -\overline{u_{l} v_{m}}
\end{array}\right) .
\end{gathered}
$$

Block 2 has $l \times m$ dimensions and block 4 has $m \times l$. Blocks 3,7 are generated by $\partial_{u} \partial_{\bar{w}}, \partial_{w} \partial_{\bar{u}}$, respectively, and are given by:

$$
\begin{gathered}
\mathbf{3}=\left(\begin{array}{cccc}
u_{1} w_{1} & u_{1} w_{2} & \cdots & u_{1} w_{p} \\
u_{2} w_{1} & u_{2} w_{2} & \cdots & u_{2} w_{p} \\
\vdots & \vdots & \ddots & \vdots \\
u_{l} w_{1} & u_{l} w_{2} & \cdots & u_{l} w_{p}
\end{array}\right), \\
\mathbf{7}=\left(\begin{array}{cccc}
\overline{u_{1} w_{1}} & \overline{u_{2} w_{1}} & \cdots & \overline{u_{l} w_{1}} \\
\overline{u_{1} w_{2}} & \overline{u_{2} w_{2}} & \cdots & \overline{u_{l} w_{2}} \\
\vdots & \vdots & \ddots & \vdots \\
\overline{u_{1} w_{p}} & \overline{u_{2} w_{p}} & \cdots & \overline{u_{l} w_{p}}
\end{array}\right) .
\end{gathered}
$$

Block 3 has $l \times p$ dimensions and block 7 has $p \times l$. Finally, blocks $\mathbf{6}, 8$ are generated by $\partial_{v} \partial_{\bar{w}}, \partial_{w} \partial_{\bar{v}}$, respectively, and are given by:

$$
\begin{gathered}
\mathbf{6}=\left(\begin{array}{cccc}
\bar{v}_{1} w_{1} & \bar{v}_{1} w_{2} & \cdots & \bar{v}_{1} w_{p} \\
\bar{v}_{2} w_{1} & \bar{v}_{2} w_{2} & \cdots & \bar{v}_{2} w_{p} \\
\vdots & \vdots & \ddots & \vdots \\
\bar{v}_{m} w_{1} & \bar{v}_{m} w_{2} & \cdots & \bar{v}_{m} w_{p}
\end{array}\right), \\
\mathbf{8}=\left(\begin{array}{cccc}
v_{1} \bar{w}_{1} & v_{2} \bar{w}_{1} & \cdots & v_{m} \bar{w}_{1} \\
v_{1} \bar{w}_{2} & v_{2} \bar{w}_{2} & \cdots & v_{m} \bar{w}_{2} \\
\vdots & \vdots & \ddots & \vdots \\
v_{1} \bar{w}_{p} & v_{2} \bar{w}_{p} & \cdots & v_{m} \bar{w}_{p}
\end{array}\right) .
\end{gathered}
$$

Block $\mathbf{6}$ has $m \times p$ dimensions and block 8 has $p \times m$. Note that when we paste all blocks together, we obtain a Hermitian matrix, so the form $\mathcal{F}$ is generally pseudo-Hermitian 
(or Hermitian if it fulfills the positive-definite condition if there are not hybrid or inverted coordinates).

By definition, a metric is a nondegenerate form, so the determinant of its matrix must not vanish. Let us see that this is satisfied for this general form $\mathcal{F}$ only if a very restrictive condition is satisfied for the number of hybrid coordinates: there must not be more than one coordinate of this kind $(l \leqslant 1)$. The determinant of a matrix can be calculated decomposing it in minors; if we consider more than one hybrid coordinate $(l>1)$, we can always reduce the original matrix determinant to sufficiently small minors which cancel all of them. This happens because only minors which include $F$ terms (those ones which appear in the main diagonal of blocks 5,9 ) contribute, any other minors are null, and if we have $l>1$ we can always reduce the determinant to minors with no $F$ terms (we are not considering the $F^{-2}$ common factor). This is a very restrictive result and forces us to consider only two cases: $l=0$ or $l=1$.

Before studying two cases separately, we can give a general expression for the determinant of $\mathcal{F}$ when $l \leq 1$ :

$$
\operatorname{det} \mathcal{F}=\frac{(-1)^{l+p}\left|u_{1}\right|^{2}+(-1)^{l+p} \delta_{l 0}\left(1-\left|u_{1}\right|^{2}\right)}{F^{n+1}},
$$

i.e., for the case without hybrid coordinates: $\operatorname{det} \mathcal{F}_{l=}$ $0=(-1)^{p} / F^{n+1}$, whereas for one hybrid coordinate: $\operatorname{det} \mathcal{F}_{l=1}=(-1)^{p+1}\left|u_{1}\right|^{2} / F^{n+1}$.

\section{B. No hybrid coordinates $(l=0)$}

When we studied the introduction of one inverted coordinate in Sec. III A, we dealt with a logarithmic argument $F$ given by Eq. (22) with $l=0, m=n-1$, $p=1$. We discussed that it was possible to obtain the metric as the induced of an Euclidean one in $\mathbb{C}^{n+1}$. For this general case, we can proceed in an analogous way. Let us define the space $M^{(+)}$as:

$M^{(+)}=\left\{Z \in \mathbb{C}^{n+1}: \sum_{i=1}^{m+1}\left|V_{i}\right|^{2}-\sum_{j=1}^{p}\left|W_{j}\right|^{2}=1\right\}$,

where we have written $Z \in \mathbb{C}^{n+1}$ as $Z=(V, W)$, with $V \in \mathbb{C}^{m+1}, W \in \mathbb{C}^{p}$. We note that $\exists k \in[1, m+1]: Z_{k}=$ $V_{k} \neq 0$ because $\sum_{i=1}^{m+1}\left|V_{i}\right|^{2}=1+\sum_{j=1}^{p}\left|W_{j}\right|^{2}>0$. We can now consider the $n$ dimensional complex manifold $N$ given by the quotient space $N^{(+)}=M^{(+)} / U(1)$. This manifold can be covered by the union of sets $\cup_{k} U_{k}$, where $U_{k}$ is given by:

$U_{k}\left(V_{k} \neq 0\right)=\left\{z \in \mathbb{C}^{n}: \sum_{\substack{i=1 \\ i \neq k}}^{m+1}\left|v_{i}\right|^{2}-\sum_{j=1}^{p}\left|w_{j}\right|^{2}>-1\right\}$, where we have introduced new coordinates $v_{i}=$ $V_{i} / V_{k}, w_{j}=W_{j} / V_{k}$. The map $\pi^{(+)}: M^{(+)}(V, W) \rightarrow$ $N^{(+)}(v, w)$ induces, from the Euclidean metric $f=$ $\sum_{i=1}^{m+1}\left|d V_{i}\right|^{2}-\sum_{j=1}^{p}\left|d W_{j}\right|^{2}$, the metric we are interested in. It is easy to conclude the Einstein character of the metric by taking into account Eq. (6). We have again a pseudo-Kähler-Einstein metric with $\lambda_{r}=n+1:$ Ric $=$ $(n+1) \mathcal{F}_{l=0}$. To show that it has also associated a constant bisectional curvature, we can use the same argument as in simpler cases: if the isometry group acts transitively on the manifold $N^{(+)}$, it suffices to show the relation at the point $(v, w)=0$. The proof of the relation in $(v, w)=0$ is similar to previous cases and the condition $\left.R_{i j \bar{k} \bar{l}}\right|_{(v, w)=0}=$ $\left.\left(\mathcal{F}_{i \bar{j}} \mathcal{F}_{k \bar{l}}+\mathcal{F}_{i \bar{l}} \mathcal{F}_{k \bar{j}}\right)\right|_{(v, w)=0, l=0}$ is satisfied. We only need to find the group which allows to generalize the result. Consider the set of matrices which act on $\mathbb{C}^{n+1}$ and preserve the Euclidean form $f\left(Z_{1}, Z_{2}\right)=\sum_{i=1}^{m+1} \overline{V_{1 i}} V_{2 i}-$ $\sum_{j=1}^{p} \bar{W}_{1 j} W_{2 j}$, where we have used the separation $Z=(V, W)$. This set forms the group called the indefinite special unitary group $S U(p, m+1)$. This group acts on $N^{(+)}$as an isometry and transitively, so the proof is completed. $\mathcal{F}$ has positive constant bisectional curvature: $R_{i \bar{j} k \bar{l}}=\left.\left(\mathcal{F}_{i \bar{j}} \mathcal{F}_{k \bar{l}}+\mathcal{F}_{i \bar{l}} \mathcal{F}_{k \bar{j}}\right)\right|_{l=0}\left(\lambda_{c}=1\right)$.

\section{One hybrid coordinate $(l=1)$}

We have previously studied a metric obtained from a form $F$ with $l=1, m=n-1, p=0$ in Sec. III B. In this case, we shall discuss a generalize form $F$ which also contains inverted coordinates: $l=1, m+p=n-1$.

When studying the simpler case $p=0$, the metric only contained terms as the ones contained in blocks 1, 5, 2, 4 . In the general case, there are terms related to every block. However, $l=1$, so block $\mathbf{1}$ reduces to one term $\mathbf{1}=-\left|u_{1}\right|^{2}$, blocks $\mathbf{4}$ and $\mathbf{7}$ reduce to vectors, and blocks $\mathbf{2}$ and $\mathbf{3}$ to their conjugate transposed ones. In any case, by using Eq. (6) is easy to obtain: $\operatorname{Ric}=(n+1) \mathcal{F}_{l=1}$. So, in it is also a pseudo-Kähler-Einstein metric in this general case.

However, it does not have associated a constant bisectional curvature. We can consider again the subspace given by $v_{i}=w_{j}=0 \forall i \in[1, m], \forall j \in[1, p]$. It is enough to show that the constant bisectional curvature relation does not hold in this subspace to complete the proof. For this general case $m+p=n-1$, the same product of metrics relation as in the simpler case $p=0$ is satisfied: $\left(\mathcal{F}_{i \bar{j}} \mathcal{F}_{k \bar{l}}+\right.$ $\left.\mathcal{F}_{i \bar{l}} \mathcal{F}_{k \bar{j}}\right)\left.\right|_{v_{i}=w_{j}=0, l=1}=\left(\delta_{i j} \delta_{k l}+\delta_{i l} \delta_{k j}\right) / F_{l=1}^{2}\left(v_{i}=w_{j}=0\right)$, where all indices are associated with direct coordinates $i, j, k, l \in[1, m]$. It also holds if all indices are associated with inverted coordinates $i, j, k, l \in[1, p]$. However, when calculating the components of the Riemann tensor, the hybrid coordinate appears explicitly in calculations through the contraction between the metric and its first derivatives. Therefore, if we calculate components $R_{i \bar{j} k \bar{l}}$ with all indices associated to direct coordinates $i, j, k, l \in[1, m]$, an extra 
term not given by the double derivative of the metric contributes. The double derivative gives a contribution equal to the product of metrics, but the contraction between the metric and its first derivatives adds a negative term of the form $-\delta_{i l} \delta_{k j} / F_{l=1}^{2}\left(v_{i}=w_{j}=0\right)$. With this, the components of the Riemann tensor in this subspace are given by $\left.R_{i \bar{j} k \bar{l}}\right|_{v_{i}=w_{j}=0}=\delta_{i j} \delta_{k l} / F_{l=1}^{2}\left(v_{i}=w_{j}=0\right)$ and do not satisfy the constant bisectional curvature relation. For terms of the Riemann tensor associated to inverted coordinates $i, j, k, l \in[1, p]$ we obtain the same expression and, therefore, we can conclude that this type of metric does not have a constant bisectional curvature.

\section{SIGNATURES OF INVERTED AND HYBRID COORDINATES}

We have discussed how the introduction of new nondirect coordinates to the logarithmic Käler potential (22) allows us to define new pseudo-Kähler-Einstein metrics non-positivedefinite. In this section we would like to clarify that these new coordinates (inverted and hybrid) have associated opposite signatures to the direct coordinates.

First of all, since we are dealing with metrics which are all Hermitian or pseudo-Hermitian, they can be locally diagonalized by an unitary matrix and their eigenvalues are all real [16]. This is particularly useful because the signature of the metrics is completely determined by the sign of their eigenvalues.

\section{A. One hybrid and arbitrary number of direct coordinates $(l=1, p=0)$}

Consider the metric $\mathcal{H}_{\mu \bar{\nu}}(20)$ introduced in Sec. III B or, analogously, the metric corresponding to the form (22) with $l=1, p=0$. The eigenvalues $\lambda$ are solutions of the equation $\operatorname{det}(\mathcal{H}-\lambda \mathbb{1})=0$, where $\mathcal{H}$ is the matrix (21). By subtracting the $H^{-2}$ factor, all eigenvalues must satisfy:

$$
(\lambda-H)^{n-2}\left[\lambda^{2}-\left(1+\frac{1}{2}\left(z_{1}-\bar{z}_{1}\right)^{2}\right) \lambda-\left|z_{1}\right|^{2} H\right]=0,
$$

where $H$ and the hybrid coordinate $z_{1}$ were introduced in Sec. III B. This equation has $n$ real roots. $n-2$ of them are $H$ and the other two are the solutions to the second degree equation between square brackets:

$$
\lambda_{ \pm}=\frac{B_{A} \pm \sqrt{B_{A}^{2}+4\left|z_{1}\right|^{2} H}}{2} .
$$

Here $B_{A}=1+\left(z_{1}-\bar{z}_{1}\right)^{2} / 2$. We find a positive root $\lambda_{+}$ and a negative one $\lambda_{-}$which, together with the other ones, complete the $n$ eigenvalues. Note that $H$ must be positive for $\log H$ to be well defined. Therefore, the signature of the metric $\mathcal{H}_{\mu \bar{\nu}}(20)$ is $(-,+,+, \ldots+)$. The minus signature corresponds to the introduction of the hybrid coordinate, whereas the positive signatures are provided by the presence of the direct ones.

On the other hand, the computation of the eigenvalues allow us to check the value of the determinant of the metric. Taking now into account the $\mathrm{H}^{-2}$ factor into the multiplication of the $n$ eigenvalues, we find:

$$
\operatorname{det} \mathcal{H}=\frac{H^{n-2} \lambda_{+} \lambda_{-}}{H^{2 n}}=-\frac{\left|z_{1}\right|^{2}}{H^{n+1}},
$$

which agrees with the result presented in Sec. III B.

\section{B. One hybrid and arbitrary number of inverted coordinates $(l=1, m=0)$}

We now consider the metric (23) corresponding to the logarithmic argument (22) when $l=1, m=0$. Again, to find the eigenvalues $\lambda$, we need to solve the equation $\operatorname{det}(\mathcal{F}-\lambda \mathbb{1})=0$, where $\mathcal{F}$ is the matrix (23) (we again do not take into account the multiplicative factor $F^{-2}$ of matrix $\mathcal{F}$ when calculating the eigenvalues). The eigenvalues equation reads:

$(\lambda+F)^{n-2}\left[\lambda^{2}+\left(1+\frac{1}{2}\left(u_{1}+\bar{u}_{1}\right)^{2}\right) \lambda+\left|u_{1}\right|^{2} F\right]=0$,

where $F$ and the hybrid coordinate $u_{1}$ were introduced in Sec. IVA. Therefore, $n-2$ eigenvalues are $-F$ and the other two correspond also to the roots of a second degree equation, which in this case reads:

$$
\lambda_{-}^{(1,2)}=\frac{-B_{B} \pm \sqrt{B_{B}^{2}-4\left|u_{1}\right|^{2} F}}{2},
$$

where $B_{B}=1+\left(u_{1}+\bar{u}_{1}\right)^{2} / 2$. In this case, it is easy to conclude that the last two eigenvalues, $\lambda_{-}^{(1)}$, and $\lambda_{-}^{(2)}$, are also negative. Note that the manifold under study is limited by the domain where $F$ is positive. Therefore, the signature of this metric with $l=1, m=0$ is negative-definite: $(-,-,-, \ldots .-)$. So, by changing the sign of the entire metric, we can define a positive-definite Kähler-Einstein geometry. We shall discuss this question in the next section.

We can again calculate the determinant as the product of the eigenvalues. Taking into account the multiplicative $F^{-2}$ factor of matrix $\mathcal{F}$, we find:

$$
\operatorname{det} \mathcal{F}=\frac{(-1)^{n-2} F^{n-2} \lambda_{-}^{(1)} \lambda_{-}^{(2)}}{F^{2 n}}=(-1)^{n} \frac{\left|u_{1}\right|^{2}}{F^{n+1}},
$$

which agrees with the result presented in Eq. (33) in Sec. IVA with $l=1, m=0$.

\section{One hybrid, one direct and one inverted coordinate $(l=1, m=1, p=1)$}

For a general case, the computation of the eigenvalues is not so simple. We can illustrate the general situation by 
considering the metric corresponding to the argument (22) with $l=1, m=1$, and $p=1$. Proceeding in the same way than in previous Secs. VA and V B, the eigenvalues $\lambda$ are determined by the equation $\operatorname{det}(\mathcal{F}-\lambda \mathbb{1})=0$, where $\mathcal{F}$ is the matrix (23). We again omit the $F^{-2}$ factor of matrix $\mathcal{F}$ when calculating the eigenvalues. Such equation reads:

$$
\lambda^{3}+A \lambda^{2}-C F \lambda-\left|u_{1}\right|^{2} F^{2}=0,
$$

where $A=\left|u_{1}\right|^{2}+\left|v_{1}\right|^{2}+\left|w_{1}\right|^{2}, \quad C=1+\left(u_{1}^{2}+\bar{u}_{1}^{2}\right) / 2$, and $F$ and coordinates $u_{1}, v_{1}, w_{1}$ were introduced in Sec. IV A. The solutions of this equation are not as trivial as the ones discussed above, but we can analyze the sign of the roots using the Descartes rule. Let us note that $A$ and $F$ are positive, whereas the sign of $C$ is not completely determined. There are regions where it is positive and regions where it is negative. In spite of this, the number of sign changes in the coefficients of the equation is always one. It ensures the existence of only one real positive root. The other two roots could be two different real negative ones, one real negative root of multiplicity two or two complex roots (one complex and its conjugate). However, this last possibility is discarded because of the Hermitian character of the metric, which ensures the presence of real roots, as we noted at the beginning of Sec. V. Therefore, we can conclude that the signature of the metric is $(-,+,-)$. As in previous Cases Secs. VA and V B, the introduction of the hybrid coordinate $u_{1}$ is associated to a negative signature. In the same way, the inverted coordinate provides a negative signature, in opposition to the direct one.

On the other hand, the Vieta formulas allow us to calculate again the determinant as the product of the eigenvalues. Taking into account the multiplicative $F^{-2}$ factor of matrix $\mathcal{F}$, we find:

$$
\operatorname{det} \mathcal{F}=\frac{\lambda_{1} \lambda_{2} \lambda_{3}}{F^{6}}=\frac{\left|u_{1}\right|^{2}}{F^{4}},
$$

where $\lambda_{1}, \lambda_{2}, \lambda_{3}$ are the solutions to the eigenvalue Eq. (42). This result agrees with the general result presented in Eq. (33) with $l=1, m=1, p=1$. It agrees also with the general statement claimed at the beginning of this section. Hybrid and inverted coordinates are associated with negative signatures, whereas direct coordinates provide positive signatures.

\section{NEGATIVE CURVATURES}

In the previous sections (except for Sec. II F), we have worked only with pseudo-Kälher-Einstein geometries with positive curvatures. This is due to our convention for defining the metric: $\mathcal{F}_{\mu \bar{\nu}}=\partial_{\mu} \partial_{\bar{\nu}} \log F$, in terms of the argument $F$ given by Eq. (22). It is easy to conclude that the definition $\mathcal{F}_{\mu \bar{\nu}}^{(-)}=-\partial_{\mu} \partial_{\bar{\nu}} \log (2-F)$ provides a pseudoKälher-Einstein metric with negative curvature. Written in that way, direct and hybrid coordinates are associated with positive signatures, whereas inverted coordinates are related to negative signatures.

\section{A. Negative bisectional curvatures}

We can illustrate this fact by analyzing cases with constant bisectional curvatures. When discussing general metrics with no hybrid coordinates in Secs. III A and IV B, we only found metrics with constant positive bisectional curvature despite of the appearance of inverted coordinates. We now discuss how to construct metrics with constant negative bisectional curvatures. Consider the space $M^{(-)}$ given by:

$M^{(-)}=\left\{Z \in \mathbb{C}^{n+1}: \sum_{i=1}^{m}\left|V_{i}\right|^{2}-\sum_{j=1}^{p+1}\left|W_{j}\right|^{2}=-1\right\}$,

where we have written $Z \in \mathbb{C}^{n+1}$ as $Z=(V, W)$, with $V \in \mathbb{C}^{m}, W \in \mathbb{C}^{p+1}$, and $m+p=n$. We have only reversed the sign of one side of the equality with respect to the definition of the space $M^{(+)}$given by Eq. (34) in the previous case with positive curvature. We also note that $\exists j \in[1, p+1]: Z_{j}=W_{j} \neq 0 \quad$ because $\quad \sum_{j=1}^{p+1}\left|W_{j}\right|^{2}=$ $1+\sum_{i=1}^{m}\left|V_{i}\right|^{2}>0$. We can now consider the $n$ dimensional complex manifold $N^{(-)}$given by the quotient space $N^{(-)}=M^{(-)} / U(1)$. This manifold can be covered by the union of sets $\cup_{k} U_{k}$, where $U_{k}$ is given by:

$U_{k}\left(W_{k} \neq 0\right)=\left\{z \in \mathbb{C}^{n}: \sum_{i=1}^{m}\left|v_{i}\right|^{2}-\sum_{\substack{j=1 \\ j \neq k}}^{p}\left|w_{j}\right|^{2}<1\right\}$,

where we have introduced new coordinates $v_{i}=V_{i} / W_{k}$, $w_{j}=W_{j} / W_{k}$. The map $\pi^{(-)}: M^{(-)}(V, W) \rightarrow N^{(-)}(v, w)$ induces, from the Euclidean metric $f=\sum_{i=1}^{m}\left|d V_{i}\right|^{2}-$ $\sum_{j=1}^{p+1}\left|d W_{j}\right|^{2}$, a metric on $N^{(-)}$given by:

$$
\mathcal{F}_{\mu \bar{\nu}}^{(-)}=-\partial_{\mu} \partial_{\bar{\nu}} \log \left(1-\sum_{i=1}^{m}\left|v_{i}\right|^{2}+\sum_{\substack{j=1 \\ j \neq k}}^{p+1}\left|w_{j}\right|^{2}\right) .
$$

The analysis of this metric is similar to the analysis of the induced metric made in the previous case for the space $M^{(+)}$, defined by Eq. (34). However, in this case, the metric has a negative constant bisectional curvature with $\lambda_{r}=-(n+1)$, and $\lambda_{c}=-1:$ Ric $=-(n+1) \mathcal{F}^{(-)}$, and $R_{i \bar{j} k \bar{l}}=-\left(\mathcal{F}_{i \bar{j}}^{(-)} \mathcal{F}_{k \bar{l}}^{(-)}+\mathcal{F}_{i \bar{l}}^{(-)} \mathcal{F}_{k \bar{j}}^{(-)}\right)$. Note that we have only changed the definition of the space $M^{(-)}$with respect to the space $M^{(+)}$, by reversing the sign of one side of the equality, that defines it in Eq. (34); the metric $f$ from which we induce the new one $\mathcal{F}^{(-)}$has not been modified. However, written as in Eq. (46), the $v_{i}$ (direct) coordinates 
have associated positive signatures, whereas the $w_{j}$ (inverted) coordinates provide negative signatures.

For instance, the particular case $p=0$ corresponds to the unit ball geometry introduced in Sec. II F. On the other hand, for the case $p=1$, the space $N^{(-)}$may be identified as the $n$ dimensional complex anti-de Sitter space [15].

\section{CONCLUSIONS}

Metrics that are solutions of vacuum Einstein field equations with cosmological constants are known as Einstein geometries. In real structures, they have played a fundamental role not only in theoretical developments but also in cosmological applications. In this work we have analyzed Einstein metrics in complex manifolds by performing a systematic study of quadratic contributions to the logarithmic Kähler potential. After reviewing well-known examples as the Fubini-Study metric or the Unit ball geometry, we have found new Kähler-Einstein and pseudo-Kähler-Einstein metrics by the introduction not only of direct coordinates, but also inverted and hybrid coordinates.

The square modulus of direct coordinates contributes positively to the argument function $F$ of the logarithmic Kähler potential. The square modulus of inverted coordinates contributes negatively to the same argument $F$. On the contrary, a hybrid coordinate is characterized by an opposite contribution of the square of its real part with respect to the contribution of the square of its imaginary part. The signature of direct coordinates is positive, whereas the signature of inverted coordinates is negative. In contrast, the signature of hybrid coordinates depends on the curvature of the metric. For positive curvatures, their signature is negative, whereas it is positive for negative curvatures.

We have found a very restrictive condition for a pseudoKähler-Einstein metric to be nondegenerate: It must have associated no more than one hybrid coordinate. This has allowed us to separate the most general cases into two different classes: Metrics with one hybrid coordinate and an arbitrary number of direct and inverted coordinates; and metrics with no hybrid coordinates and also an arbitrary number of direct and inverted coordinates. There are important differences between these two types of geometries. The latter one not only corresponds to KählerEinstein or pseudo-Kähler-Einstein metrics, but also these geometries are characterized by a constant bisectional curvature. This is not the case of the former one, whose geometries own less isometries. In any case, we have built explicit examples of both types, where it is easy to check our general results associated with different geometrical features, such as determinants or curvatures.

\section{ACKNOWLEDGMENTS}

This work was partially supported by the MICINN (Ministerio de Ciencia e Innovación, Spain) Project No. PID2019-107394GB-I00 (AEI/FEDER, UE). J. A. R. C. acknowledges support by Institut Pascal at Université ParisSaclay during the Paris-Saclay Astroparticle Symposium 2021, with the support of the P2IO (Physics of the two infinities and Origins) Laboratory of Excellence (program "Investissements d'avenir" ANR-11-IDEX-0003-01 ParisSaclay and ANR-10-LABX-0038), the P2I (Physics of the two infinities) axis of the Graduate School Physics of Université Paris-Saclay, as well as IJCLab (Laboratoire de Physique des 2 Infinis Irène Joliot-Curie), CEA (Commissariat à l'énergie atomique), IPhT (Institute of Theoretical Physics), APPEC (Astroparticle physics Consortium), the IN2P3 (Institut national de physique nucléaire et de physique des particules) master projet UCMN and EuCAPT (European Consortium for Astroparticle Theory). This research was supported by the Munich Institute for Astro- and Particle Physics (MIAPP) which is funded by the Deutsche Forschungsgemeinschaft (DFG, German Research Foundation) under Germany's Excellence Strategy_EXC-2094-390783311.
[1] S. Deser and B. Zumino, A complete action for the spinning string, Phys. Lett. B 65, 369 (1976).

[2] S.-T. Yau, On the Ricci curvature of a compact Kähler manifold and the complex Monge-Ampére equation, I, Commun. Pure Appl. Math. 31, 339 (1978).

[3] W. de Sitter, On the relativity of inertia: Remarks concerning Einstein's latest hypothesis, Proc. Kon. Ned. Acad. Wet. 19, 1217 (1917).

[4] W. de Sitter, On the curvature of space, Proc. Kon. Ned. Acad. Wet. 20, 229 (1917).
[5] J. M. Maldacena, The large N limit of superconformal field theories and supergravity, Adv. Theor. Math. Phys. 2, 231 (1998).

[6] M. Nakahara, Geometry, Topology and Physics (Taylor and Francis, London, 2003).

[7] G. Tian, Canonical Metrics in Kähler Geometry (ETH Zürich Birkhäuser Verlag, Basel, Switzerland, 2000).

[8] A. Newlander and L. Nirenberg, Complex analytic coordinates in almost complex manifolds, Ann. Math. 65, 391 (1957). 
[9] A. Moroianu, Lectures on Kähler Geometry (Cambridge University Press, Cambridge, 2007), Vol. 69.

[10] D. Huybrechts, Complex Geometry: An Introduction (Springer, Berlin, 2005).

[11] B. Yang and F. Zheng, On curvature tensors of hermitian manifolds, Commun. Anal. Geom. 26, 1195 (2018).

[12] K.-F. Liu and X.-K. Yang, Geometry of hermitian manifolds, Int. J. Math. 23, 1250055 (2012).
[13] K. Liu and X. Yang, Ricci curvatures on Hermitian manifolds, Trans. Am. Math. Soc. 369, 5157 (2017).

[14] S. Kobayashi and K. Nomizu, Fundations of Differential Geometry (Wiley, New York, 1969), Vol. 2.

[15] A. Ben-Ahmed and A. Zeghib, On homogeneous HermiteLorentz spaces, Asian J. Math. 20, 531 (2016).

[16] S. Roman, Advanced Linear Algebra (Springer, New York, 2008). 